
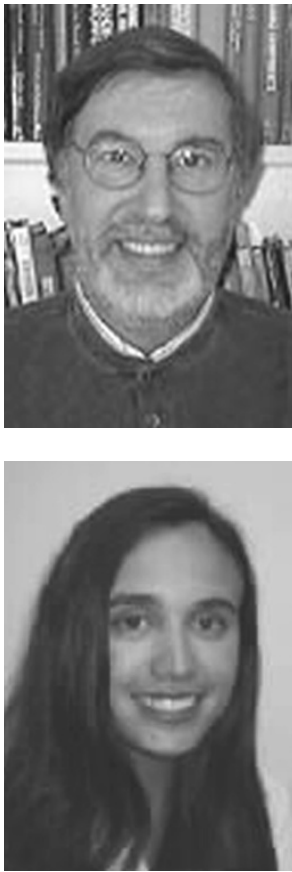

\title{
Modelling the Wildlife
} Conservation Society database: Saving money for saving tigers (and gorillas and jaguars and elephants ....)

Received: 4th April, 2001

\section{Richard Colombo}

is an Associate Professor of Marketing at Fordham University Graduate School of Business. He has also taught at New York University, Columbia University and Yale University. Before becoming an academic, he worked in The Social Survey Division of The Office of National Statistics. He has an MA (Physics) from Cambridge University, UK and an MBA and PhD (Marketing) from Columbia University. His main research interests are in the analysis of marketing data.

\section{Dina Mele}

is Assistant Director of Membership and has been with the Wildlife Conservation Society (WCS) for just over four years. In her tenure, the WCS Membership base has grown from about 80,000 to approximately 105,000 households. She is responsible for all direct mail efforts and fund-raising initiatives via the web and oversees all data entry and fulfillment as well as the onsite membership sales programme at the Bronx Zoo, NY Aquarium, Central Park, Queens and Prospect Park Wildlife Centers. Before joining WCS, Dina worked in the children's publishing industry and holds a BA in English and History. She is currently finishing the MBA programme at Fordham University with a concentration in Marketing and Management.

Abstract Not-for-profit organisations, or those with relatively small databases, are often unable to test fund-raising or other types of direct marketing appeals. In these circumstances, a good model of the organisation's database can greatly increase net revenues. This paper compares several methods of modelling a database using the Wildlife Conservation Society database as an example. A new model for estimating potential donation amounts seems to offer some practical as well as conceptual improvements over existing procedures.

\section{INTRODUCTION}

Charitable organisations share many of the same problems as catalogue mailers and other direct marketers. They have to devise good ways of maintaining their database of members and donors and of exploiting the information contained in

Richard Colombo Graduate School of Business, Fordham University, 113 W 60 Street, New York, NY 10023, USA.

Tel: +1(212) 636 6380;

e-mail:

richard.colombo@verizon.net their database. But they generally do not have the resources at their disposal that large direct marketers can command and, in particular, are rarely able to test a fund-raising solicitation. Even if they had the resources, the size of their databases, which are usually quite a bit smaller than those of for-profit direct marketers, would make testing a rather futile activity. Consequently, for many charitable organisations, exploitation of the organisation's database for fund-raising efforts is often, a rather ad hoc affair. This is not to say that charitable organisations are not efficient and effective direct marketers - often experience, intuition and managerial judgment can be highly effective — but rather that their approach of necessity 
lacks a scientific, open basis that makes it difficult to learn systematically about how fund-raising efforts might be improved.

This paper reports on a statistical investigation of the Wildlife Conservation Society (WCS) popularly known as the Bronx Zoo database of members and contributors. The purpose of the investigation was to build a model that WCS could use for targeting potential donors. Expectations were that the model would be an improvement over current procedures and that it would help WCS better understand the drivers of response. Another expected benefit of the modelling approach was that it would be easier and faster to choose potential donors for targeting than the existing, somewhat ad hoc procedures.

Direct marketers have used models of one kind or another for targeting for half a century or more. Models based on scoring, frequency and monetary value (RFM) variables are widely used but increasingly, more sophisticated approaches such as linear regression, logistic regression, tree regression (eg CART, CHAID), probit, discriminant analysis and neural networks have been called into service; see, for example, Shepherd et al. ${ }^{1}$ for an elementary introduction to a variety of RFM models and Lilien and Rangaswarmy ${ }^{2}$ for examples of logistic regression and neural networks.

Regression models of one kind or another are the dominant approach to ranking a database in terms of good and poor prospects. Most of the regression models reported in the literature focus on predicting response. For single-shot mailings involving just one product, such as a book or a magazine subscription, predicting whether or not someone will respond is fine. But for the WCS, where the gift can take any positive value, it makes sense to target low probability individuals if they have potentially high gift amounts. While regression approaches can be used to predict gift amount, an alternative approach is to build a stochastic model of gift-giving behaviour. Stochastic models have not much application in the database marketing literature. Some exceptions are Schmittlein and Petersen, ${ }^{3}$ Rao and Steckel ${ }^{4}$ and Colombo and Jiang. ${ }^{5}$ In the next section some background to the WCS is given. In the following section, a variety of different models are fitted to a sample of records from the WCS database. Then a stochastic model is developed for the amount of a gift and how the model can be used to rank a database is shown. The paper concludes with a discussion.

\section{BACKGROUND TO WCS}

The WCS is a non-profit conservation organisation headquartered at the Bronx Zoo. It conducts over 300 conservation projects in more than 50 countries and runs the largest network of urban zoological parks in the world including the Bronx Zoo, NY Aquarium, Central Park, Queen and Prospect Park Wildlife Centers. Major funding comes from private donors, including major donors, mid-level and low-level donors, or 'members'. Currently there are about 100,000 member households made up of two distinct groups: Metro members (90 per cent who join for free admission to the zoo and consist mostly of families in the New York Tristate area and National members (10 per cent who believe in the conservation mission and are located all over the USA.

All donors are entered into the WCS database, through a system called Raiser's Edge. The database holds approximately 250,000 records of current and lapsed members and donors. Data collected include: name, address, phone, 
Table 1: Recency, frequency and monetary value segments

\begin{tabular}{ll}
\hline Metro members & National members \\
\hline Contribute to add-a-gift & New this year \\
\$25-\$75 appeals, last three years & $\$ 10-£ 24$ appeals \\
New, family plus + & $\$ 25-\$ 75$ appeals \\
New, not family level & Non-members, $\$ 25-\$ 75$ appeals \\
Lapsed, appeals three plus years & Three plus years, no appeals \\
$\$ 75+$ to appeals & Lapsed, $\$ 25-\$ 75$ appeals \\
\hline
\end{tabular}

solicitation history, giving history and membership or donor type.

Three appeals a year are sent to select members to raise money for international funds, Targeting and segmentation is based on a rudimentary recency, frequency and monetary model (RFM) and historical performance. The RFM segments used by WCS are shown in Table 1.

\section{RESPONSE MODELLING}

The main aim of building a predictive model of response is to generate more funds for international conservation programmes by better targeting donors. The model ranks the records in the database from most promising to least promising and allows a cut-off to be chosen below which records are not selected. Usually the ranked database is grouped into deciles which are chosen for targeting as long as they are expected to deliver some positive contribution.

The quality of the ranking depends critically on the variables in the database. The variables in the database were:

- response (1 or 0$)$

— first gift amount

- days in database

— last gift amount

- recency

— total amount of gifts

— total number of gifts

- average gift

- current member

- have they ever given to area appeals
- have they ever given general conservation gifts

- have they ever given to mega vertebrates

- have they ever given to other animals

- have they ever given to other holiday appeals?

Leeflang et al. ${ }^{6}$ point out that there are indirect benefits to building models.

These are:

- improved understanding

- problem finding

- use of information

— framework for discussion

- reallocation of management time

- pin-point changes in the environment.

These indirect benefits will be revisited in the final section. Leeflang et al. also point out some implementation criteria for models:

- models should be simple

- models should be built in an evolutionary way

- models should be complete on important issues

- models should be adaptive

- models should be robust.

These criteria have been kept in mind in building the predictive model. These issues will be taken up more fully in the final section.

Several response models were constructed using a sample from the 
Table 2: Coefficients of logistic regression model

\begin{tabular}{lrlr}
\hline & Value & Std. error & $\boldsymbol{t}$ value \\
\hline (Intercept) & -0.691 & 0.2558 & -2.70 \\
Recency & -0.001 & 0.0003 & -4.25 \\
Average gift & -0.035 & 0.0064 & -5.43 \\
Area & 0.529 & 0.1110 & 4.76 \\
Mega & 0.470 & 0.1096 & 4.29 \\
Other & 0.339 & 0.1654 & 2.05 \\
\hline
\end{tabular}

WCS database to predict the probability that someone would respond and the response amount. Logistic regression, a generalised additive model (gam) and tree regression were used in the investigation. In addition, a stochastic model for amount of response was developed and tested. The sample from the WCS database consisted of almost 6,000 records drawn randomly from the lists of three previous appeals - for tigers, gorillas and jaguars. There were about 2,000 sample records for each appeal. The resulting database was randomly split into two samples, one for estimation and the other for validation.

To conform to usual practice, a frequency variable was constructed using the total number of gifts and the number of days in database.

The first model fitted was a conventional logistic regression model to predict the response probability. First, all variables were included in the model and then a reduced model was fitted with only the significant variables retained. (A stepwise logistic regression produced the same final model). Only the recency, average gift amount, and whether contributed to area, mega vertebrates and other animal appeals variables were found to contribute significantly to response. The signs of the coefficients were as expected with the possible exception for average gift amount where the higher amounts give rise to lower predicted response. This, of course, highlights the importance of modelling not just response, since higher gift amounts could compensate for lower response probabilities for deciding whether someone should be targeted.

The logistic regression is linear in the predictor variables. To examine whether there were non-linearities in the recency, frequency and average gift amount a generalised additive model $^{7}$ was also fitted. Generalised additive models have the general form

$$
\eta(x)=\alpha+f_{1}\left(x_{1}\right)+f_{2}\left(x_{2}\right)+\ldots+f_{p}\left(x_{p}\right)
$$

where each of the $x_{i}$ are predictors and the $f_{i}$ are smooth functions of the predictors chosen from a particular class and are fitted to the data using a non-parametric technique. Smoothing splines with five degrees of freedom were chosen as the class of smooth functions. The model fitted was then a non-parametric regression model of the form

$$
\log \left(\frac{\pi(x)}{1-\pi(x)}\right)=\eta(x)
$$

There was little difference in the fit of the two models as measured by the deviance - a measure of goodness-of-fit - and in terms of their predictions on the validation sample. There were, however, some mild non-linear effects. As can be seen in Figure 1, smoothing does little for the recency variable so one might just as well use recency in its raw form. It seems that the same cannot be said for the frequency variable. The flat part of the curve, however, fits the bulk of the data well and the downward sloping part seems only necessary to fit a few observations. Since the curve is essentially flat for the bulk of the data, it can be seen why the frequency variable was not significant in the logistic regression. Finally, the average gift amount does seem to exhibit some 

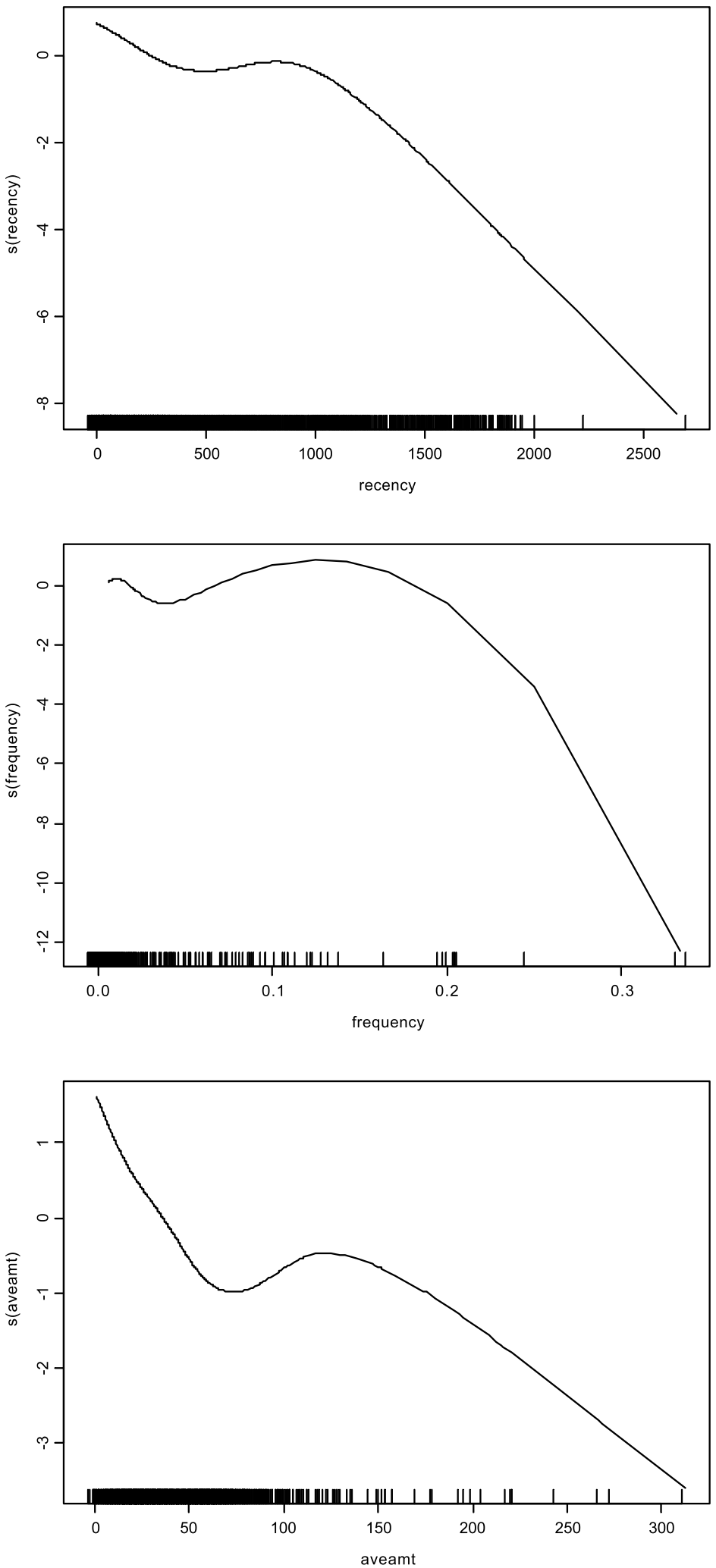

Figure 1 


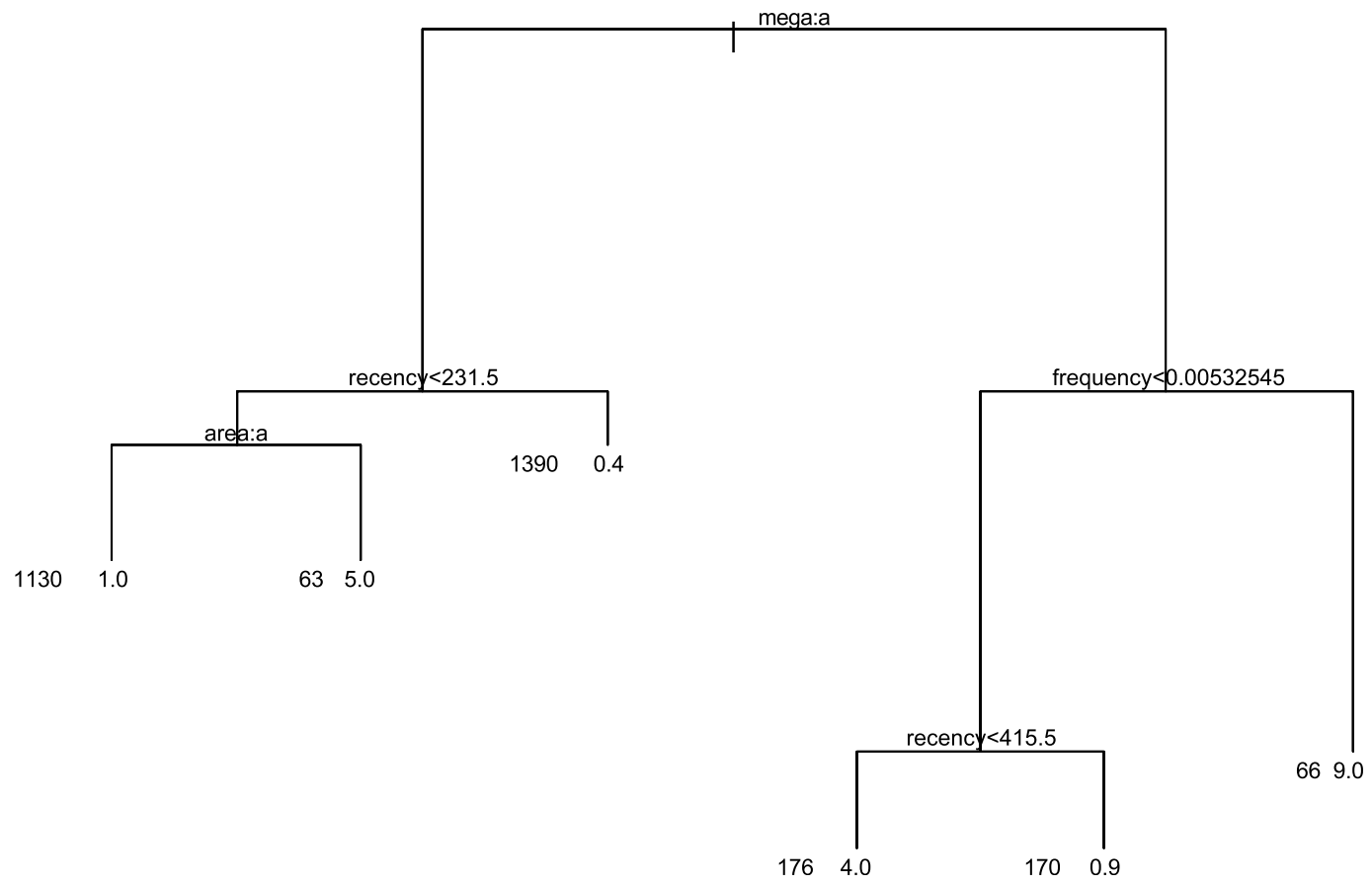

Figure 2

non-linearity. What the plot suggests is that response is inversely related to average gift amount in the range $\$ 0-\$ 100$ and $\$ 150+$ but that for those individuals who give on average between $\$ 100$ and $\$ 150$ the response probability is positively related to amount. While the generalised additive model provides some additional insight into the data, for predictive purposes it offers no significant improvement over the more familiar logistic regression.

Both the usual logistic regression and the non-parametric logistic regression are additive models in that they do not include interaction terms among the predictors, although interaction terms can be included in such models. Tree-based regression models (eg Breiman et al. ${ }^{8}$ Haughton and Oulabi ${ }^{9}$ ) are more readily able to capture non-additive behaviour. Instead of estimating a regression tree with response as the dependent variable, the amount of the gift was used as the dependent variable. (If an individual did not respond to the appeal, their gift was $\$ 0)$. The tree model was fitted by binary recursive partitioning which splits the dataset into a hierarchy of increasingly homogeneous subsets. The fitting of trees is not as straightforward as linear or logistic regression because the fitting algorithm usually grows an overly complex tree. It is then necessary to 'prune' the tree to produce a more parsimonious tree. Figure 2 shows the result of the fitting and pruning procedure.

The numbers at the bottom of the tree in Figure 2 show the number of cases falling into each group and the predicted contribution for an individual belonging to the group estimated from the validation sample. For example, 1,130 individuals (out of 2,996 in the validation sample) who had not contributed to a 'mega-vertebrate' or an area appeal in the past but who had 
Table 3: Decile analysis for five models

\begin{tabular}{|c|c|c|c|c|c|c|c|c|c|c|}
\hline \multirow[b]{2}{*}{ decile } & \multicolumn{2}{|c|}{ Additive } & \multicolumn{2}{|c|}{ Logistic } & \multicolumn{2}{|c|}{ Tree } & \multicolumn{2}{|c|}{$\begin{array}{l}\text { Logistic with } \\
\text { amount }\end{array}$} & \multicolumn{2}{|c|}{$\begin{array}{l}\text { Logistic with } \\
\text { 'shrunk' amount }\end{array}$} \\
\hline & obs \% & pred \% & obs \% & pred \% & obs $\$$ & pred \$ & obs \$ & pred \$ & obs \$ & pred \$ \\
\hline 1 & 15.0 & 18.0 & 16.3 & 18.0 & 1,617 & 1,514 & 1,664 & 1,263 & 1,604 & 1,342 \\
\hline 2 & 7.0 & 6.7 & 6.0 & 6.3 & 35 & 317 & 430 & 458 & 407 & 531 \\
\hline 3 & 4.0 & 4.3 & 3.0 & 4.2 & 27 & 293 & 236 & 326 & 236 & 361 \\
\hline 4 & 1.7 & 3.2 & 3.3 & 3.2 & 338 & 293 & 363 & 289 & 287 & 308 \\
\hline 5 & 3.0 & 2.4 & 1.0 & 2.5 & 357 & 289 & 76 & 263 & 230 & 274 \\
\hline 6 & 2.3 & 1.8 & 2.3 & 2.0 & 141 & 193 & 124 & 241 & 110 & 243 \\
\hline 7 & 0.3 & 1.4 & 1.3 & 1.6 & 343 & 123 & 58 & 216 & 96 & 214 \\
\hline 8 & 1.0 & 1.2 & 0.3 & 1.3 & 241 & 123 & 166 & 187 & 122 & 188 \\
\hline 9 & 0.7 & 0.9 & 1.3 & 1.1 & 241 & 123 & 208 & 135 & 75 & 153 \\
\hline 10 & 1.7 & 0.4 & 1.7 & 0.6 & 339 & 123 & 354 & 56 & 513 & 73 \\
\hline
\end{tabular}

made a gift within the past 231.5 days gave an average gift of $\$ 1$. If it costs $\$ 1$ to mail a solicitation, then only three groups should be mailed determined by the following rules:

— if $($ mega $=1)$ and (frequency $>0.005325)$ then mail

- if (mega $=1)$ and (frequency $<0.005325)$ and (recency $<415.5)$ then mail

- if $($ mega $=0)$ and (recency $<231.5)$ and $($ area $=1)$ then mail.

These rules result in targeting 10 per cent $(=305 / 2996)$ of the file to generate about $\$ 7,500$ in gifts for a cost of about $\$ 300$.

The regression tree methodology is appealing because it focuses directly on the key variable, the expected amount of the gift, and not just on the response probability. It also results in simple rules to identify who to target. The performance of the regression tree as judged by cross-validation is, however, inferior to the logistic regression and the additive logistic regression models. This is consistent with the experiences of others.

The results of the logistic and non-parametric regression and tree regression are summarised in Table 3 . Each of the three models was fitted to the estimation sample and the resulting model was used to predict response in the validation sample. The predicted responses were then used to rank the validation sample and to form deciles. Thus, the non-parametric logistic regression or additive model predicted that the top 10 per cent of the sample would have a response rate of 18 per cent. In fact the response was 15 per cent. The logistic regression is treated in the same way. And the tree regression predicted that $\$ 1,617$ in gifts would come from the top decile when in fact $\$ 1,514$ were received. Also included is the result of ranking the responses by the predicted probabilities from the logistic regression multiplied by the average gift amount. The last two columns refer to a model that will be discussed in the following section, 'The Monetary Value Distribution'.

Some comments on the table:

- although the results above are presented with response grouped into deciles, the models provide a predicted response for each individual record. There is therefore no need to categorise records into deciles for purposes of targeting. Records where the expected gift (= probability $X$ average gift amount) exceeds the cost of the solicitation should be selected

- in all cases, there appears to be a 'blip' in the tenth decile where there 
is a greater response than predicted. This is due to a small number of first-time donors who were targeted and responded. Such donors would not have had time to contribute to more than one major appeal and therefore would have been assigned a low probability of response (see Table 2 for coefficients of logistic regression)

- the two models that predict gift amounts seem less able to order deciles from high to low amounts. (Actually, there is probably little to choose between a response-only model and a model that takes into account gift amount as can be seen from Figure 4). In part, this is because the predictor variables are really measuring latent variables which are measured with error and which have different precisions. For example, someone who contributed once and gave $\$ 100$ has an average gift amount of $\$ 100$. But to believe that this is what they will give in the future would be rather rash since there is rather little information on which to base a prediction. On the other hand, there would be a fair bit of confidence that someone who had given 10 times, and each time gave $\$ 100$, would be likely to give $\$ 100$ next time because there is now considerably more information. In the next section, a model that takes the amount of information into account is described briefly.

\section{THE MONETARY VALUE DISTRIBUTION}

Following Colombo and Jiang, ${ }^{10}$ it is assumed that each individual donor donates an amount that can vary from occasion to occasion around some 'true' or expected value. Since the donation, $z$, must be a positive amount, it is assumed that it is distributed as a gamma

distribution given by

$$
g(z ; u, \theta)=\frac{\theta^{u}}{\Gamma(u)} z^{u-1} e^{-\theta z}
$$

The mean is $u / \theta$. The model allows different donors to have different mean donation amounts by assuming that the mean has a distribution of values in the population. To simplify matters a little however, it is assumed that $u$ is fixed and $\theta$ varies, which is equivalent to assuming that all donors have the same coefficient of variation. It is then assumed that $\theta$ has a gamma distribution indexed with the parameters $v$ and $\theta$

$$
h(\theta ; v, \phi)=\frac{\phi^{v}}{\Gamma(v)} \theta^{\nu-1} e^{-\phi \theta}
$$

From (1) and (2), the distribution of observed donations for an individual can be found by integrating out $\theta$ so that the following is obtained

$$
\begin{aligned}
f\left(z_{1}, \ldots z_{k} \mid u, \theta\right) & =f\left(z_{1} \mid u, \theta\right) \ldots f\left(z_{k} \mid u, \theta\right) \\
f\left(z_{1}, \ldots z_{k} \mid u, v, \phi\right) & =\int_{0}^{\infty} \frac{\theta^{k u}}{\Gamma(u)^{k}} \\
\left(z_{1} \ldots z_{k}\right)^{u-1} e^{-\theta k \bar{z}} \frac{\phi^{v}}{\Gamma(v)} \theta^{\nu-1} e^{-\theta \phi} d \theta & (3) \\
f\left(z_{1}, \ldots z_{k} \mid u, v, \phi\right) & =\frac{\Gamma(k u+v)}{\Gamma(u)^{k} \Gamma(v)} \\
\left(z_{1} \ldots z_{k}\right)^{u-1} & \left(\frac{\phi}{k \bar{z}+\phi}\right)^{v}\left(\frac{1}{k \bar{z}+\phi}\right)^{k u}
\end{aligned}
$$

Now using (3), an expression can be derived for the expected value of an individual's contribution given their past contributions. Suppose that an individual has made $k$ donations of $z_{1}, z_{2}, \ldots z_{k}$. The expected value of this individual's 'true' donation, that is the amount they would be expected to donate next time, 


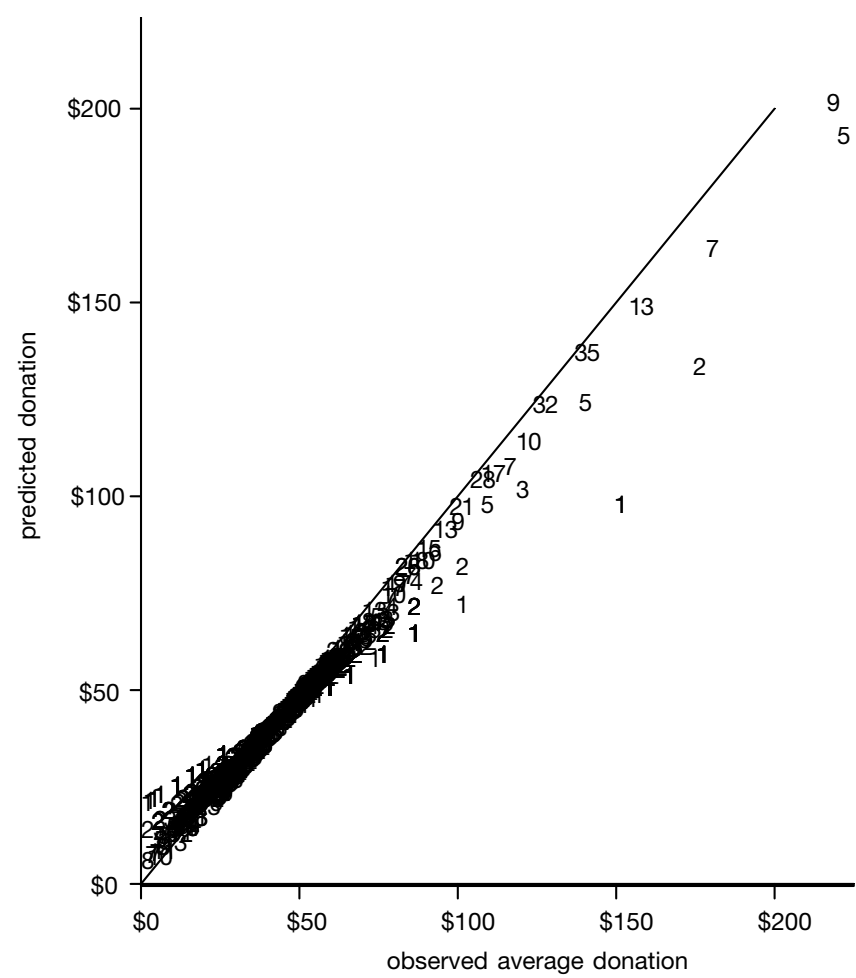

Figure 3: Predicted and observed donations (numbers in plot show number of donations)

can be derived by an application of Bayes' Theorem (see foot of page).

The parameters $u, v$ and $\phi$ can be estimated using (3) and the expected donation amount given an individual's past history can be calculated from (4).

The population mean of donations, $m$, of (3) is $\phi u /(v-1)$ and substituting this into (4) by eliminating $\phi$ gives

$$
\frac{k u \bar{z}+m(v-1)}{k u+(v-1)}
$$

which can more usefully be written as

$$
\begin{aligned}
& \lambda \bar{z}+(1-\lambda) m \\
& \lambda=\frac{k u}{k u+v-1}
\end{aligned}
$$

If the number of past donations, $k$, for an individual is high then $\lambda$ will be close to 1 and the expected donation for that individual will be close to the individual's mean. On the other hand, if $k$ is small that individual's expected donation would

$$
\begin{aligned}
E\left(\frac{u}{\theta} \mid z_{1}, z_{2, \ldots} z_{k}\right)= & \frac{\int_{0}^{\infty}\left(\frac{u}{\theta}\right)\left(\frac{\theta^{u}}{\Gamma(u)}\right)^{k}\left(z_{1}, z_{2}, \ldots z_{k}\right)^{u-1} e^{-\theta k \bar{z}} \frac{\theta}{\Gamma(v)} \theta^{\nu-1} e^{-k \phi} d \theta}{\int_{0}^{\infty}\left(\frac{\theta^{u}}{\Gamma(u)}\right)^{k}\left(z_{1}, z_{2}, \ldots z_{k}\right)^{u-1} e^{-\theta k \bar{z} \frac{\theta^{\nu}}{\Gamma(v)}} \theta^{\nu-1} e^{-k \phi} d \theta} \\
& =\frac{u(k \bar{z}+\phi)}{u k+v-1}
\end{aligned}
$$




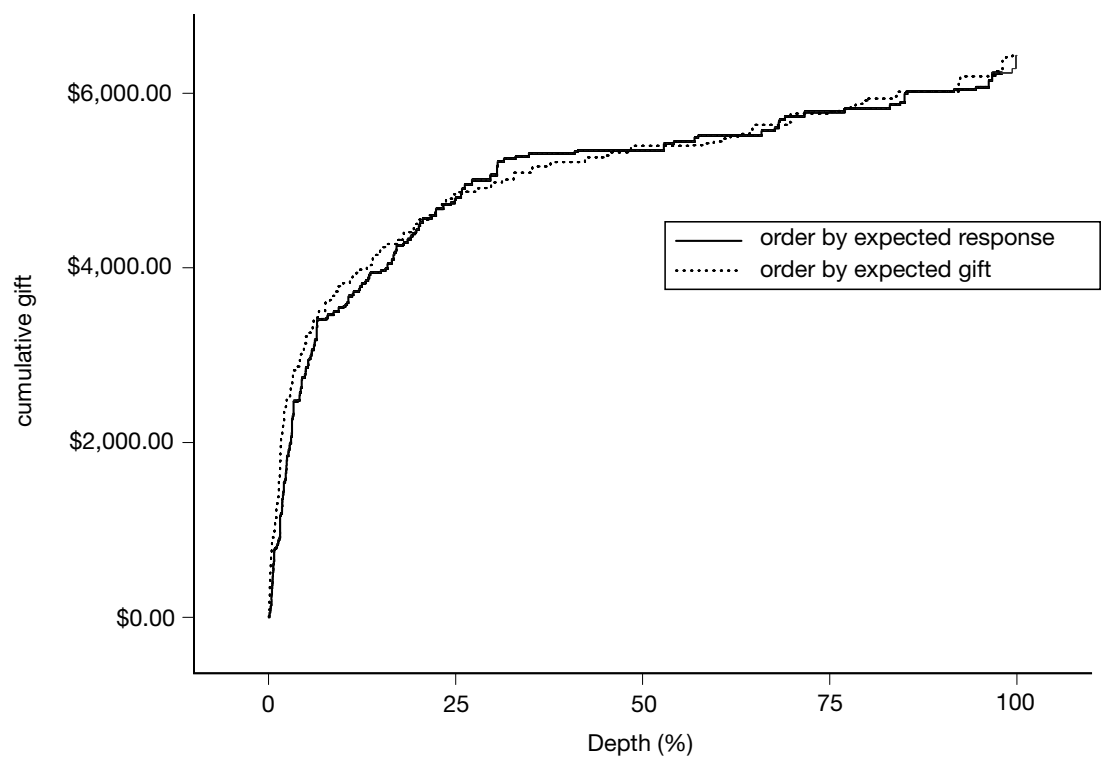

Figure 4: Revenue from mailing using expected gift or expected response for ordering the database

be estimated by something close to the overall mean. Similarly, if $v$ is large, which means that the population distribution is tightly clustered around the population mean, or $u$ is small, which means that the variance of the individual-level distribution is high, the expected donation would be close to $m$ as would be expected. The expression in (5) is a shrinkage estimator because it shrinks individual estimates to the overall mean by an amount that depends on the precision of individual estimates.

Since the estimation sample did not have the full transaction history, the first and last gift amounts were used to estimate the parameters. Values of $u=3.49, v=4.26$ and $\phi=38.5$ were obtained. A plot in Figure 3 of expected values given by (5) and the observed values shows the expected regression-to-the-mean effect. The plot shows more regression-to-the-mean where the number of gifts is small.

The question of deciding how deep to go in the ranked database is now addressed. It is assumed that the donation, $p$, and the probability of donating, $\Pi$, are known. Then all records where $p_{i} \Pi_{i}>c_{i}$ would be selected. To a good approximation, this will maximise profits. (However, if $p$ and $\Pi$ have a probability distribution, the profit maximising cut-off is not so easily determined).

More insight into this question can be obtained by plotting a gains chart that shows cumulative response against cumulative numbers mailed. As Figure 4 shows, overall contribution stabilises at about a depth of 40 per cent.

Finally, because WCS is not able to test response rates for a fund-raising effort, it is difficult to decide on the optimal depth, which depends on response rate. Figure 5 shows how the optimal depth varies with mailing costs for a given response rate. Similar curves can be constructed for various response rates, thus allowing WCS to choose a depth that seems reasonable and robust even when there is uncertainty in response rates.

\section{DISCUSSION}

It is often difficult for non-profit organisations to test a proposed mail-out. In such cases the relationship between response and the demographic or 


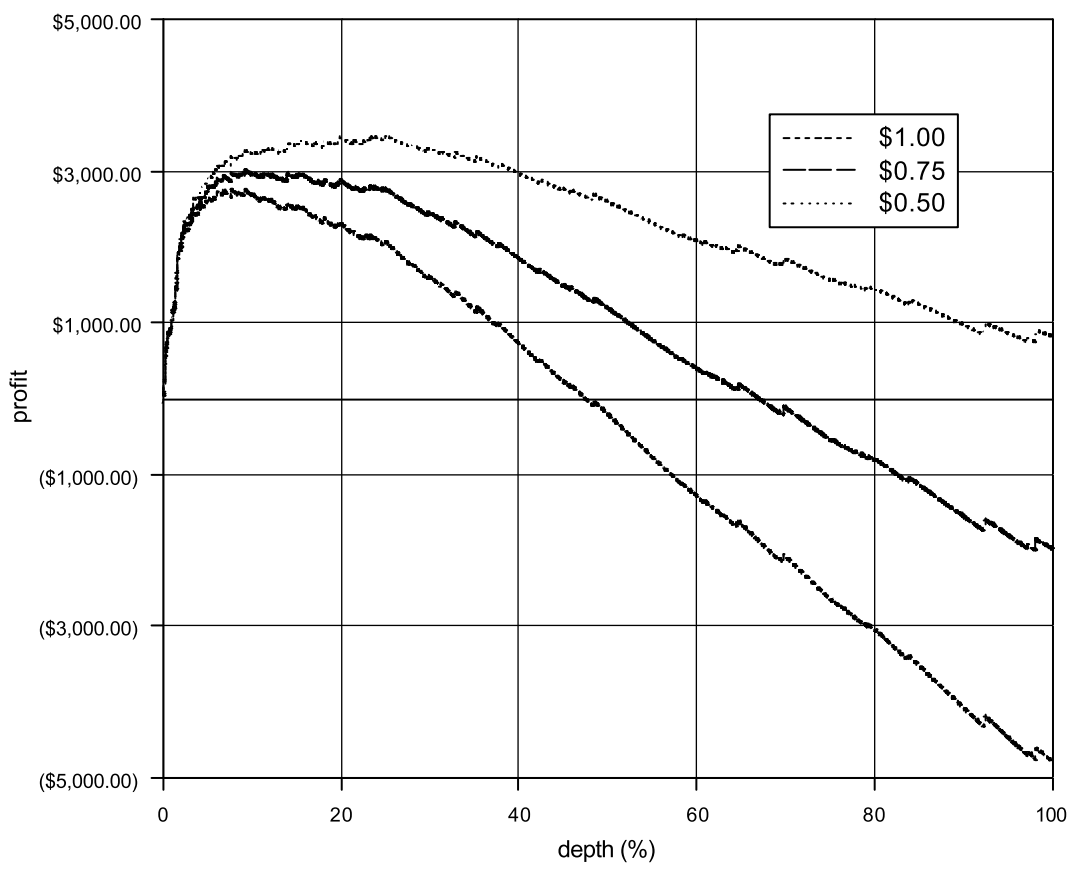

Figure 5: Profit curves for various unit mailing costs

behavioural variables needs to be established from the transaction history stored in the database. Traditional RFM scoring methods rank prospects from good to poor but they do not provide predictions of the probability or amount of response, whereas statistical approaches give predictions that can be used for deciding which members of the database should be contacted.

But a good statistical model has benefits that go beyond this. The experience at WCS is that the models were easier to implement than traditional RFM procedures. They also generated useful discussions about how to manage WCS's relationship with its members and donors. This was because in developing the models it was recognised that they were to be used by non-statisticians. Further work is now under way to find a model that is largely independent of the nature of the appeal and which adapts to changing circumstances while at the same time being robust to idiosyncratic conditions. Model building is a continuing process. But this is as it should be; for the model should represent management's latest and best view of how members of the database respond.

\section{References}

1 Shepherd, D. (1990) 'The new direct marketing', Business One Irwin, Homewood, IL.

2 Lilien, G. and Rangaswamy, A. (1998) 'Marketing engineering', Addison Wesley.

3 Schmittlein, D. C. and Peterson, R. A. (1994) 'Customer base analysis: An industrial purchase process application', Marketing Science, Vol. 13, pp. 41-67.

4 Rao, V. R. and Steckel, J. H. (1995) 'Selecting, evaluating, and updating prospects in direct mail marketing', Journal of Direct Marketing, Vol. 9, No. 2, pp. 20-31.

5 Colombo, R. and Jiang, W. (1999) 'A stochastic RFM model', Journal of Interactive Marketing, Summer.

6 Leeflang et al. (2000) 'Building models for marketing decisions', Kluwer Academic Publishers, Dordrecht, The Netherlands.

7 Hastie, T. and Tibshirani, R. (1990) 'Generalized additive models', Chapman \& Hall, London.

8 Breiman, L., Friedman, J., Olshen, R. and Stone, C. (1984) 'Classification and regression trees'.

9 Haughton, D. and Oulabi, S. (1993) 'Direct Marketing Modelling with CART and CHAID', Journal of Direct Marketing, Vol. 7 (Summer), pp. 16-26, Chapman \& Hall, New York.

10 Colombo and Jiang (1999) op. cit. 